\title{
On the Security of CAMELLIA against the Square Attack
}

\author{
Yongjin Yeom, Sangwoo Park, and Iljun Kim \\ National Security Research Institute \\ 161 Gajeong-dong, Yuseong-gu, Daejeon, 305-350 Korea \\ \{yjyeom, psw\}@etri.re.kr, ijkim@dingo.etri.re.kr
}

\begin{abstract}
Camellia is a 128 bit block cipher proposed by NTT and Mitsubishi. We discuss the security of Camellia against the square attack. We find a 4 round distinguisher and construct a basic square attack. We can attack 5 round Camellia by guessing one byte subkey and using $2^{16}$ chosen plaintexts. Cosidering the key schdule, we may extend this attack up to 9 round Camellia including the first $\mathrm{FL} / \mathrm{FL}^{-1}$ function layer.
\end{abstract}

\section{Introduction}

Camellia [5] is a 128-bit block cipher which was announced by NTT and Mitsubishi in 2000. It has the modified Feistel structure with irregular rounds, so called the $F L / F L^{-1}$ function layer. The round function is based on that of the block cipher E2 [13] by NTT whereas the $F L / F L^{-1}$ layer comes from MISTY[18] by Mitsubishi. Camellia was submitted to the standardization and the evaluation projects such as ISO/IEC JTC 1/SC 27, CRYPTREC, and NESSIE. Recently, Camellia was selected as an algorithm for the second phase of the NESSIE project.

Currently, the most efficient methods analyzing Camellia are truncated differential cryptanalysis and higher order differential attack. Kanda and Matsumoto 12] studied the security against truncated differential cryptanalysis from the designer's standpoint. They found the upper bound of the best bytewise characteristic probability and proved that Camellia with more than 11 rounds are secure against truncated differential cryptanalysis. Most analyses on Camellia consider simplified version without $F L / F L^{-1}$ function layers. For instance, S. Lee et al.15] attacked eight round Camellia using truncated differential cryptanalysis. M. Sugita et al.[19] found a nontrivial 9 round bytewise characteristics and a seven round impossible differential for Camellia. Kawabata and Kaneko[11] showed that Camellia can be attacked by higher order differential attack up to 10 rounds. Some other analyses can be found in [5].

The square attack was a dedicated attack on the block cipher SQUARE[6] and applied to block ciphers of the SPN structure such as Rijndael[7.816], CRYPTON [9], and Hierocrypt [3]. In order to apply the square attack on the Feistel structure, Lucks[17] introduced the saturation attack, as a variation of the square attack. He analyzed the Twofish algorithm of the modified Feistel 
structure. Recently, Y. He and S. Qing[10] showed that six round Camellia are breakable by square attack.

In this paper, we apply the square attack to Camellia including $F L / F L^{-1}$ function layers. We suggest the basic attack which breaks 5 round Camellia using $2^{16}$ chosen plaintexts and $2^{8}$ key guessings. Also, the key schedule is considered so that the square attack on 256 bit Camellia is faster than exhaustive key search up to 9 rounds.

Section 2 briefly describes the structure of Camellia. A basic attack based on a 4 round distinguisher is given in Section 3 and extensions of the basic attack up to 9 round Camellia is proposed in Section 4.

\section{Description of the Camellia}

Camellia has a 128 bit block size and supports 128, 192 and 256 bit keys. The design of Camellia is based on the Feistel structure and its number of rounds is 18 (128 bit key) or 24(192, 256 bit key). The $F L / F L^{-1}$ function layer is inserted at every 6 rounds in order to thwart future unknown attacks. Before the first round and after the last round, there are pre- and post-whitening layers which use bitwise exclusive-or operations with 128 bit subkeys, respectively.

One round substitution and permutation structure is adopted as the round function $F$. Let $X_{L}^{(r)}$ and $X_{R}^{(r)}$ be the left and the right halves of the $r$ round inputs, respectively, and $k^{(r)}$ be the $r$ round subkey. Then the Feistel structure of Camellia can be written as

$$
\begin{aligned}
& X_{L}^{(r+1)}=X_{R}^{(r)} \oplus F\left(X_{L}^{(r)}, k^{(r)}\right), \\
& X_{R}^{(r+1)}=X_{L}^{(r)} .
\end{aligned}
$$

In the following substitution $S$, the four types of S-boxes $s_{1}, s_{2}, s_{3}$, and $s_{4}$ are used. Each of them is affinely equivalent to an inversion over $G F\left(2^{8}\right)$. Actually, $s_{2}, s_{3}, s_{4}$ are variations of $s_{1}$. The only property of S-boxes used for the square attack is that they are one-to-one functions. The substitution function $S:\{0,1\}^{64} \rightarrow\{0,1\}^{64}$ which consists of S-boxes is also a one-to-one function defined by

$$
\left(x_{1}, \ldots, x_{8}\right) \stackrel{S}{\longrightarrow}\left(s_{1}\left(x_{1}\right), s_{2}\left(x_{2}\right), s_{3}\left(x_{3}\right), s_{4}\left(x_{4}\right), s_{2}\left(x_{5}\right), s_{3}\left(x_{6}\right), s_{4}\left(x_{7}\right), s_{1}\left(x_{8}\right)\right) .
$$

The permutation function $P:\{0,1\}^{64} \rightarrow\{0,1\}^{64} \operatorname{maps}\left(z_{1}, \ldots, z_{8}\right)$ to $\left(z_{1}^{\prime}, \ldots, z_{8}^{\prime}\right)$ defined by

$$
\begin{aligned}
& z_{1}^{\prime}=z_{1} \oplus z_{3} \oplus z_{4} \oplus z_{6} \oplus z_{7} \oplus z_{8}, \\
& z_{2}^{\prime}=z_{1} \oplus z_{2} \oplus z_{4} \oplus z_{5} \oplus z_{7} \oplus z_{8}, \\
& z_{3}^{\prime}=z_{1} \oplus z_{2} \oplus z_{3} \oplus z_{5} \oplus z_{6} \oplus z_{8}, \\
& z_{4}^{\prime}=z_{2} \oplus z_{3} \oplus z_{4} \oplus z_{5} \oplus z_{6} \oplus z_{7}, \\
& z_{5}^{\prime}=z_{1} \oplus z_{2} \oplus z_{6} \oplus z_{7} \oplus z_{8},
\end{aligned}
$$




$$
\begin{aligned}
& z_{6}^{\prime}=z_{2} \oplus z_{3} \oplus z_{5} \oplus z_{7} \oplus z_{8}, \\
& z_{7}^{\prime}=z_{3} \oplus z_{4} \oplus z_{5} \oplus z_{6} \oplus z_{8}, \\
& z_{8}^{\prime}=z_{1} \oplus z_{4} \oplus z_{5} \oplus z_{6} \oplus z_{7} .
\end{aligned}
$$

We can also express the function $P$ in the matrix form:

$$
\left(\begin{array}{l}
z_{8}^{\prime} \\
z_{7}^{\prime} \\
z_{6}^{\prime} \\
z_{5}^{\prime} \\
z_{4}^{\prime} \\
z_{3}^{\prime} \\
z_{2}^{\prime} \\
z_{1}^{\prime}
\end{array}\right)=\left(\begin{array}{llllllll}
0 & 1 & 1 & 1 & 1 & 0 & 0 & 1 \\
1 & 0 & 1 & 1 & 1 & 1 & 0 & 0 \\
1 & 1 & 0 & 1 & 0 & 1 & 1 & 0 \\
1 & 1 & 1 & 0 & 0 & 0 & 1 & 1 \\
0 & 1 & 1 & 1 & 1 & 1 & 1 & 0 \\
1 & 0 & 1 & 1 & 0 & 1 & 1 & 1 \\
1 & 1 & 0 & 1 & 1 & 0 & 1 & 1 \\
1 & 1 & 1 & 0 & 1 & 1 & 0 & 1
\end{array}\right)\left(\begin{array}{l}
z_{8} \\
z_{7} \\
z_{6} \\
z_{5} \\
z_{4} \\
z_{3} \\
z_{2} \\
z_{1}
\end{array}\right) .
$$

The round function $F:\{0,1\}^{64} \times\{0,1\}^{64} \rightarrow\{0,1\}^{64}$ is defined as a composition of $S$ and $P$ functions as follows:

$$
(X, k) \stackrel{F}{\longrightarrow} P(S(X \oplus k)) .
$$

At every 6 rounds the functions $F L$ and $F L^{-1}$ are inserted. We denote bitwise-and, bitwise-or operations by $\cap, \cup$ and a $n$ bit rotation by $\lll n$. The left 64 bit half $\left(X_{L}, X_{R}\right)$ is mapped to $\left(Y_{L}, Y_{R}\right)$ by the function $F L$.

$$
\left(X_{L}\left\|X_{R}, k l_{L}\right\| k l_{R}\right) \stackrel{F L}{\longrightarrow}\left(Y_{L}, Y_{R}\right)
$$

where

$$
\begin{aligned}
& Y_{R}=\left(\left(X_{L} \cap k l_{L}\right) \lll 1\right) \oplus X_{R}, \\
& Y_{L}=\left(Y_{R} \cup k l_{R}\right) \oplus X_{L} .
\end{aligned}
$$

and the inverse $F L^{-1}$ of $F L$ is used for the right half as follows:

$$
\left(Y_{L}\left\|Y_{R}, k l_{L}\right\| k l_{R}\right) \stackrel{F L^{-1}}{\longrightarrow}\left(X_{L}, X_{R}\right)
$$

where

$$
\begin{aligned}
& X_{L}=\left(Y_{R} \cup k l_{R}\right) \oplus Y_{L}, \\
& X_{R}=\left(\left(X_{L} \cap k l_{L}\right) \lll 1\right) \oplus Y_{R} .
\end{aligned}
$$

The key schedule of Camellia will be briefly considered in Section 4 .

\section{Basic Square Attack}

The concept of the $\Lambda$-set, which was introduced by Daemen et al. [6], plays an important role in the square attack. 
Let $\mathcal{F}$ be a collection of state bytes $X=\left(x_{1}, x_{2}, \ldots, x_{n}\right)$ where $x_{i}$ is the $i$-th byte of $X$. If the $i$-th bytes of elements in $\mathcal{F}$ are different one another, the $i$-th byte is called an 'active' byte. Likewise, the $j$-th byte is 'passive' (or fixed), if the $j$-th bytes of states in $\mathcal{F}$ have the same value.

A collection $\mathcal{F}$ of 256 state bytes is called a $\Lambda$-set, if every byte of $\mathcal{F}$ is either active or passive. More precisely, if $X$ and $Y$ are arbitrary elements of a $\Lambda$-set $\mathcal{F}$, then

$$
\begin{cases}x_{i} \neq y_{i}, & \text { if the } i \text {-th byte is active, } \\ x_{i}=y_{i}, & \text { otherwise }\end{cases}
$$

where $x_{i}$ and $y_{i}$ are the $i$-th byte of $X$ and $Y$, respectively. Note that an arbitrary collection $\mathcal{F}$ has non-active and non-passive bytes in general. The $i$-th byte in a collection $\mathcal{F}$ is called balanced, if $\bigoplus_{X \in \mathcal{F}} x_{i}=0$.

The main operations of the Camellia are bitwise exclusive-or(XOR) and substitution using one-to-one $8 \times 8 \mathrm{~S}$-boxes $s_{i}$. If an active(passive) byte of a $\Lambda$-set is used as an input of S-boxes $s_{i}$, then the output is also active(passive). But the output of $s_{i}$ is not necessarily balanced when its input is balanced.

Some properties of XOR operation can be summarized as shown in Table 1

Table 1. Some properties of XOR operation

\begin{tabular}{|c||c|c|c|}
\hline XOR $(\oplus)$ & active byte & passive byte & balanced byte \\
\hline \hline active byte & balanced byte & active byte & balanced byte \\
\hline passive byte & active byte & passive byte & balanced byte \\
\hline balanced byte & balanced byte & balanced byte & balanced byte \\
\hline
\end{tabular}

\subsection{Four Round Distinguishers}

Let $X_{L}^{(r)}, X_{R}^{(r)}$ be the left and the right inputs of the $r$-th round. Then we can construct a 4 round distinguisher as follows:

Choose

$$
X_{L}^{(1)}=\left(\alpha_{1}, \alpha_{2}, \ldots, \alpha_{8}\right), \quad X_{R}^{(1)}=\left(A, \beta_{2}, \ldots, \beta_{8}\right)
$$

as a $\Lambda$-set $\mathcal{F}$ of 256 input plaintexts, where $\alpha_{i}, \beta_{j}$ are constants and $A$ is an active bytes of $\mathcal{F}$. Because $X_{L}^{(1)}$ is passive, the output of the first round function $F$ is also passive. Thus, the input of the 2 nd round can be written of the form

$$
X_{L}^{(2)}=\left(A, \gamma_{2}, \ldots, \gamma_{8}\right), \quad X_{R}^{(2)}=\left(\alpha_{1}, \alpha_{2}, \ldots, \alpha_{8}\right)
$$

where $\gamma_{i}$ are constants. In the 2 nd round, the input $X_{L}^{(2)}$ for $F$ is transformed as follows:

$$
\left(A, \gamma_{2}, \ldots, \gamma_{8}\right) \stackrel{F}{\longrightarrow}\left(B, C, D, \delta_{4}^{\prime}, E, \delta_{6}^{\prime}, \delta_{7}^{\prime}, F\right),
$$

where $B, C, D, E$, and $F$ are active. Thus, we have

$$
X_{L}^{(3)}=\left(B, C, D, \delta_{4}, E, \delta_{6}, \delta_{7}, F\right), \quad X_{R}^{(3)}=\left(A, \gamma_{2}, \ldots, \gamma_{8}\right)
$$




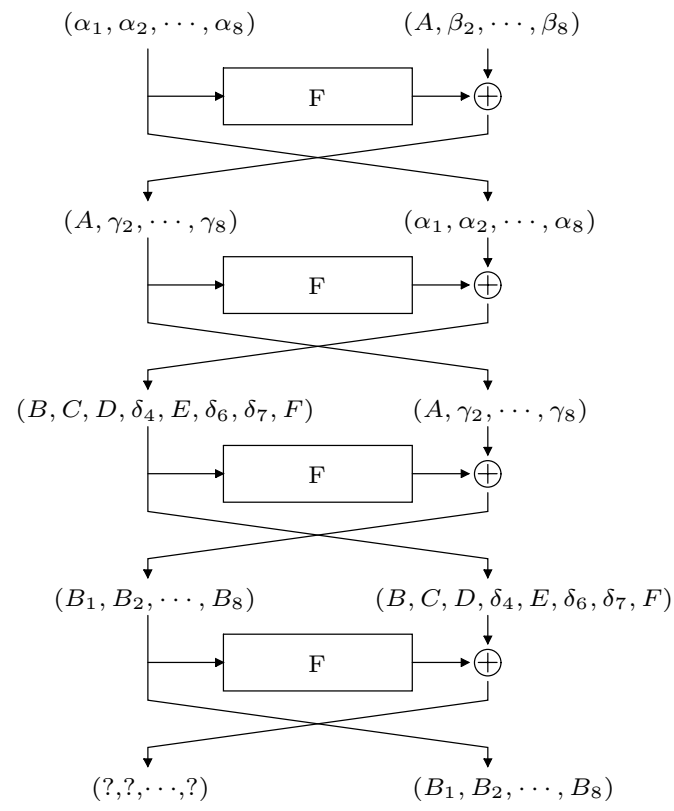

Fig. 1. A four round distinguisher

as an input for the 3rd round. Applying the 3rd round function to $X_{L}^{(3)}$, we expect that each state byte in the left half of the input for the 4th round is balanced. This implies that all bytes in the right half of the 4 th round output are balanced. Thus, we obtain a 4 round distinguisher.

Note that only 2 round functions are effectively activated in this 4 round distinguisher. This corresponds to the 2 round distinguisher for the SPN structure. If we change the position of the active byte in $X_{R}^{(1)}$, we obtain 8 different distinguishers.

\subsection{Five Round Square Attack}

From the above distinguisher, we can construct a basic square attack on the 5 round Camellia without pre- and post-whitenings.

Step 1. Guess the 1st byte $k$ of the first round key.

Step 2. As input plaintexts, choose a $\Lambda$-set $\mathcal{F}$ of the form

$$
\mathcal{F}=\left\{\left(X_{L}(i), X_{R}(i)\right) \mid 0 \leq i \leq 255\right\}
$$

where for arbitrarily chosen constants $\alpha_{i}, \beta_{j}$,

$$
\begin{aligned}
& X_{L}(i)=\left(i, \alpha_{2}, \ldots, \alpha_{8}\right), \\
& X_{R}(i)=\left(s_{1}(i \oplus k), s_{1}(i \oplus k), s_{1}(i \oplus k), \beta_{4}, s_{1}(i \oplus k), \beta_{6}, \beta_{7}, s_{1}(i \oplus k)\right) .
\end{aligned}
$$


Step 3. If $k$ is a correct key, we can expect the left half of the 2nd round inputs consists of constant states. For example, the 1st output byte $z_{1}^{\prime}$ of the 1st round function is $z_{1}^{\prime}(i)=z_{1} \oplus z_{3} \oplus z_{4} \oplus z_{6} \oplus z_{7} \oplus z_{8}$, where $z_{1}=s_{1}(i \oplus k)$ and $z_{3}, z_{4}, \ldots, z_{8}$ are constants solely depending upon $\alpha_{2}, \ldots, \alpha_{8}$. Taking exclusive-or of $z_{1}^{\prime}(i)$ and the 1 st byte $s_{1}(i \oplus k)$ of $X_{R}(i)$, we have a constant byte which is independent of $i$. Using the same argument, we can show that each byte of $X_{L}^{(2)}$ is a constant.

Step 4. The right half $X_{R}^{(2)}$ of the 2 nd round input is identical to $X_{L}^{(1)}$. Thus we can use the 4 round distinguisher previously mentioned.

Step 5. Let $C_{L}, C_{R}$ be corresponding outputs of the input $\Lambda$-set $\mathcal{F}$ which is chosen in Step 2. If all bytes of $C_{R}$ are balanced, then we can accept $k$ as the correct key. Otherwise, go to Step 1 and guess another key and repeat.

For this 5 round attack, we use $2^{8}$ times 5 round encryptions in every key guessing. A wrong key can pass the balance test with a probability $2^{-64}$, i.e. negligible. Thus, the number of plaintexts needed for this attack is $2^{8} \times 2^{8}=2^{16}$, and the same number of 5 round encryptions is required.

\subsection{Six Round Square Attack}

We can extend this basic attack to 6 round Camellia by adding a round at the beginning. The key idea for 6 round attack is to choose a collection of plaintexts whose 1 round output is a $\Lambda$-set $\mathcal{F}$ as described in (11). To do this, we assume additional 5 bytes of the first round key.

Let $k_{1}^{(2)}$ be the first byte of the second round key and $k_{i}^{(1)}$ the $i$-th byte of the 1 st round key. Suppose that we guess $k_{1}^{(1)}, k_{2}^{(1)}, k_{3}^{(1)}, k_{5}^{(1)}, k_{8}^{(1)}$, and $k_{1}^{(2)}$, correctly. Then we can find a set $\mathcal{F}^{(1)}$ of plaintexts so that the second round input is a $\Lambda$-set $\mathcal{F}^{(2)}$ of the form

$$
\mathcal{F}^{(2)}=\left\{\left(X_{L}^{(2)}(i), X_{R}^{(2)}(i)\right) \mid 0 \leq i \leq 255\right\}
$$

where

$$
\begin{aligned}
X_{L}^{(2)}(i) & =\left(i, \alpha_{2}, \ldots, \alpha_{8}\right), \\
X_{R}^{(2)}(i) & =\left(s(i), s(i), s(i), \beta_{4}, s(i), \beta_{6}, \beta_{7}, s(i)\right), \\
s(i) & =s_{1}\left(i \oplus k_{1}^{(2)}\right) .
\end{aligned}
$$

It is easy to see that the left half $X_{L}^{(1)}(i)$ of an input $\Lambda$-set

$$
\mathcal{F}^{(1)}=\left\{\left(X_{L}^{(1)}(i), X_{R}^{(1)}(i)\right) \mid 0 \leq i \leq 255\right\}
$$

should be exactly equal to $X_{R}^{(2)}(i)$ for each $i$ and the right half $X_{R}^{(1)}(i)$ of that can be determined by the subkeys $k_{1}^{(1)}, k_{2}^{(1)}, k_{3}^{(1)}, k_{5}^{(1)}$, and $k_{8}^{(1)}$. For example, the 1st output byte $z_{1}^{\prime}$ of the 1st round function can be written as 


$$
\begin{aligned}
& z_{1}^{\prime}(i)= s_{1}\left(s(i) \oplus k_{1}^{(1)}\right) \oplus s_{3}\left(s(i) \oplus k_{3}^{(1)}\right) \oplus s_{4}\left(\beta_{4} \oplus k_{4}^{(1)}\right) \oplus s_{3}\left(\beta_{6} \oplus k_{6}^{(1)}\right) \\
& \oplus s_{4}\left(\beta_{7} \oplus k_{7}^{(1)}\right) \oplus s_{1}\left(s(i) \oplus k_{8}^{(1)}\right) \\
&=s_{1}\left(s(i) \oplus k_{1}^{(1)}\right) \oplus s_{3}\left(s(i) \oplus k_{3}^{(1)}\right) \oplus s_{1}\left(s(i) \oplus k_{8}^{(1)}\right) \oplus \beta
\end{aligned}
$$

where $\beta=s_{4}\left(\beta_{4} \oplus k_{4}^{(1)}\right) \oplus s_{3}\left(\beta_{6} \oplus k_{6}^{(1)}\right) \oplus s_{4}\left(\beta_{7} \oplus k_{7}^{(1)}\right)$ is independent of $i$. Thus we can choose

$$
i \oplus s_{1}\left(s(i) \oplus k_{1}^{(1)}\right) \oplus s_{3}\left(s(i) \oplus k_{3}^{(1)}\right) \oplus s_{1}\left(s(i) \oplus k_{8}^{(1)}\right)
$$

as the first byte $S_{1}$ of $X_{R}^{(1)}(i)$ so that the first byte of $X_{L}^{(2)}(i)$ is active. Similarly, remaining bytes of $X_{R}^{(1)}(i)$ can be calculated.

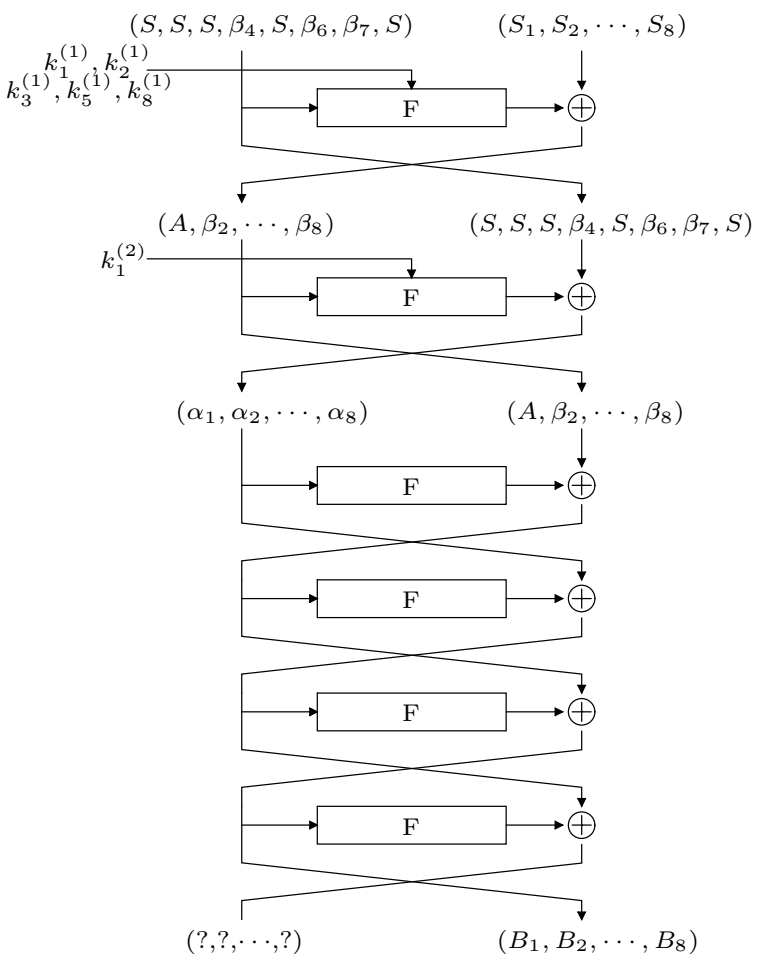

Fig. 2. A square attack on 6 round Camellia

For each $\Lambda$-set $\mathcal{F}^{(1)}$ determined by the 6 byte subkeys guessing, we can check the balance of the right half of the 6 th round output. In this check, a wrong key can be accepted with a probability $2^{-64}$. Thus, the 6 round attack requires $2^{8} \times 2^{48}$ plaintexts and $2^{48}$ subkeys guessing.

By adding a round at the end, we obtain another square attack(See 10]) on 6 round Camellia. But it makes the attack exceeding 6 rounds with the $F L / F L^{-1}$ layer much harder. 


\section{Key Schedule and Extension of the Basic Attack}

\subsection{Key Schedule of Camellia}

To extend the basic attack on over 6 round Camellia with $F L / F L^{-1}$ function layer, we consider the key schedule. The round keys are bitwise rotations of $K_{L}$, $K_{R}, K_{A}$, and $K_{B}$ which are calculated from the master keys $K_{L}$ and $K_{R}$. In this calculation, they use the reduced rounds of Camellia with a constant key. We do not describe the details here(See [1]). Table 2 shows how to select 1-10 round keys from $K_{L}, K_{R}, K_{A}$, and $K_{B}$.

Table 2. Subkeys for 192/256-bit secret key

\begin{tabular}{|c|c|c|}
\hline & subkey & value \\
\hline$F$ (Round 1) & $k^{(1)}$ & $\left(K_{B} \lll_{0}\right)_{L(64)}$ \\
\hline$F$ (Round 2) & $k^{(2)}$ & $\left(K_{B} \lll_{0}\right)_{R(64)}$ \\
\hline$F$ (Round 3) & $k^{(3)}$ & $\left(K_{R} \lll 15\right)_{L(64)}$ \\
\hline$F$ (Round 4) & $k^{(4)}$ & $\left(K_{R} \lll_{15}\right)_{R(64)}$ \\
\hline$F$ (Round 5) & $k^{(5)}$ & $\left(K_{A} \lll 15\right)_{L(64)}$ \\
\hline$F$ (Round 6) & $k^{(6)}$ & $\left(K_{A} \lll_{15}\right)_{R(64)}$ \\
\hline$F L$ & $k l_{1(64)}$ & $\left(K_{R} \lll 30\right)_{L(64)}$ \\
\hline$F L^{-1}$ & $k l_{2(64)}$ & $\left(K_{R} \lll_{30}\right)_{R(64)}$ \\
\hline$F$ (Round 7) & $k^{(7)}$ & $\left(K_{B} \lll_{30}\right)_{L(64)}$ \\
\hline$F$ (Round 8) & $k^{(8)}$ & $\left(K_{B} \lll 30\right)_{R(64)}$ \\
\hline$F$ (Round 9) & $k^{(9)}$ & $\left(K_{L} \lll 45\right)_{L(64)}$ \\
\hline$F($ Round 10$)$ & $k^{(10)}$ & $\left(K_{L} \lll 45\right)_{R(64)}$ \\
\hline
\end{tabular}

Note that seven and eight round keys $k^{(7)}$ and $k^{(8)}$ are nothing but 30 bit rotations of the first and the second round keys, respectively. This property will be used to attack more than 6 rounds of Camellia.

\subsection{An Observation on the $F L / F L^{-1}$ Layer}

Consider the reduced model of 6 round Camellia with the $F L / F L^{-1}$ layer. As mentioned previously, if we assume 6 byte subkeys correctly, every byte of the right half of the 6th round outputs is balanced. By guessing additional 7 bits of subkey $k l_{2}$, we can partially invert $F L / F L^{-1}$ layer.

Let $\left(C_{L}, C_{R}\right)$ be an output of the $F L^{-1}$ function. Then we can determine the leftmost 7 bits of the input $y_{R, 4}$ for the $F L^{-1}$ function from two bytes $x_{R, 4}$ and $x_{L, 4}$ using the relation

$$
y_{R, 4}=x_{R, 4} \oplus\left(x_{L, 4} \cap k l_{2 L, 4}\right) \lll 1,
$$

where $y_{R, 4}, x_{R, 4}, x_{L, 4}$ and $k l_{2 L, 4}$ are the fourth bytes of $Y_{R}, X_{R}, X_{L}$ and $k l_{2 L}$, respectively. 


\subsection{Square Attacks on 256 bit Camellia up to 7, 8, and 9 Rounds}

Now, we consider 256 bit Camellia and its key schedule. We can extend the previous observation on the $F L / F L^{-1}$ layer to a 7 round square attack. We should assume 7 byte subkeys $k_{1}^{(7)}, \ldots, k_{7}^{(7)}$ out of the seventh round key $k^{(7)}$ to determine two byte outputs $x_{R, 4}$ and $x_{L, 4}$ of $F L^{-1}$ function. But the 7 th round key $k^{(7)}$ is nothing but the 30 bit left rotation of the first round key $k^{(1)}$. In fact, we only guess additional 18 bits. Thus, the number of bits we need to guess for 7 round Camellia is $73=58$ (round 1,7$)+8($ round 2$)+7\left(F L^{-1}\right.$ layer $)$. When we apply the square attack to 7 round Camellia, we can check only 7 bits of the 6 th round outputs. Thus, a wrong key can pass the test with a probability $2^{-7}$. We need $11 \Lambda$-sets as input plaintexts to eliminate a wrong key. The algorithm to attack 7 round Camellia can be summarized as follows:

Step 1. Guess 6 byte subkeys $k_{1}^{(1)}, k_{2}^{(1)}, k_{3}^{(1)}, k_{5}^{(1)}, k_{8}^{(1)}$, and $k_{1}^{(2)}$ of the first and the second round.

Step 2. Prepare $11 \Lambda$-sets as plaintexts so that inputs of the third round are of the form

$$
X_{L}^{(3)}=\left(\alpha_{1}, \alpha_{2}, \ldots, \alpha_{8}\right), \quad X_{R}^{(3)}=\left(A, \beta_{2}, \ldots, \beta_{8}\right)
$$

Note that the only byte $A$ of them is active. Thus, we expect the right half of the 6th round outputs is balanced, if key guessing is correct.

Step 3. Partially decrypt outputs and test the balance of them.

3.1. Guess additional 25 bit subkeys for $F L^{-1}$ and the 7 th round.

3.2. Decrypt ciphertexts and determine 7 bits of the right half of the 6 th round outputs.

3.3. Check if this 7 bits are balanced for all $11 \Lambda$-sets.

3.4. If so, accept 73 bit subkeys as a correct key.

3.5. Otherwise, discard 25 bit subkeys guessed in Step 3.1 and choose another 25 bits. If all possible 25 bits are checked, go to Step 1 and repeat Step 2 and Step 3.

For each subkey candidate, we need to encrypt $11 \Lambda$-sets, which costs

$$
2^{48}(\text { subkeys }) \times 2^{8}(\Lambda \text {-set size }) \times 11(\text { the number of } \Lambda \text {-sets })
$$

encryptions of 7 round. Also, one round decryptions and partial invertings of $F L^{-1}$ function are needed for them with the computational complexity

$$
2^{73}(\text { subkeys }) \times 2^{8}(\Lambda \text {-set size }) \times 11(\text { the number of } \Lambda \text {-sets }) \text {. }
$$

Thus, total amount of cipher execution is approximately $2^{81.7}$ encryptions.

With helpful comments of anonymous referees, this attack could be improved as follows: for a given 6 byte subkeys of the first and the second rounds, first prepare $4 \Lambda$-sets and see whether $2^{25}$ subkeys for the $F L^{-1}$ and the 7 th round pass balanced tests. With probability $1 / 8$, one of the subkeys can pass these tests. In that case, the remaining $7 \Lambda$-sets can be exercised. This procedure reduced 
the plaintext cost to $(4+7 / 8) 2^{56}$. Also, the time complexity can be reduced to $2^{80.2}$ encryptions.

By assuming all round keys in round 8 and 9, we construct an attack algorithm on 9 round Camellia. One byte of the 8th round key is already guessed in the second round. Therefore, 193 bits of subkey guessing is needed to attack 9 round Camellia. It is of course infeasible but faster than exhaustive key search.

\section{Conclusion}

We have discussed the security of Camellia against the square attack. We have treated the reduced round Camellia without pre- and post-whitenings including the $F L / F L^{-1}$ layers. The key schedule has been considered to reduce the number of subkey guess and how to treat the $F L / F L^{-1}$ function layers has been presented.

Table 3. Summary of attacks on 256 bit Camellia

\begin{tabular}{|c||c|l|c|c|l|}
\hline \multicolumn{1}{|c||}{ Rounds } & $F L / F L^{-1}$ & Methods & Plaintexts & Time & Comments \\
\hline \hline 5 & N/A & Square Attack & $2^{10.3}$ & $2^{48}$ & He \& Qing[10](Pre-Whitening) \\
\hline 5 & N/A & Square Attack & $2^{16}$ & $2^{16}$ & This paper \\
\hline \hline 6 & N/A & Higher Order DC & $2^{17}$ & $2^{19.4}$ & Kawabata \& Kaneko[11] \\
\hline 6 & N/A & Square Attack & $2^{11.7}$ & $2^{112}$ & He \& Qing[10] (Pre-Whitening) \\
\hline 6 & N/A & Square Attack & $2^{56}$ & $2^{56}$ & This paper \\
\hline \hline 7 & $\times$ & Higher Order DC & $2^{19}$ & $2^{61.2}$ & Kawabata \& Kaneko[11] \\
\hline 7 & $\times$ & Truncated DC & $2^{82.6}$ & 192 & S. Lee et al.[15] \\
\hline 7 & $\bigcirc$ & Square Attack & $2^{58.3}$ & $2^{80.2}$ & This paper \\
\hline \hline 8 & $\times$ & Higher Order DC & $2^{20}$ & $2^{126}$ & Kawabata \& Kaneko[11] \\
\hline 8 & $\times$ & Truncated DC & $2^{83.6}$ & $2^{55.6}$ & S. Lee et al.[15] \\
\hline 8 & $\bigcirc$ & Square Attack & $2^{59.7}$ & $2^{137.6}$ & This paper \\
\hline \hline 9 & $\times$ & Higher Order DC & $2^{21}$ & $2^{190.8}$ & Kawabata \& Kaneko[11] \\
\hline 9 & $\bigcirc$ & Square Attack & $2^{60.5}$ & $2^{202.2}$ & This paper \\
\hline \hline 10 & $\times$ & Higher Order DC & $2^{21}$ & $2^{254.7}$ & Kawabata \& Kaneko[11] \\
\hline
\end{tabular}

Table 3 summarizes attacks on 256 bit Camellia by the number of rounds. Time complexities in the table is the number of encryptions.

Up to 9 rounds, the square attack is a faster way to attack Camellia than the brute force key search.

Acknowledgment. We would like to thank anonymous referees for their helpful comments and suggestions. As mentioned at the end of Section 4, we could reduce the plaintext requirement as well as time complexity according to the anonymous advice. 


\section{References}

1. K. Aoki, T. Ichikawa, M. Kanda, M. Matsui, S. Moriai, J. Nakajima and T. Tokita. Camellia: A 128-Bit Block Cipher Suitable for Multiple Platforms. Proceedings of Selected Areas in Cryptography (to appear in LNCS by Springer-Verlag) (2000) 41-54.

2. K. Aoki, T. Ichikawa, M. Kanda, M. Matsui, S. Moriai, J. Nakajima and T. Tokita. Camellia - A 128-bit Block Cipher. Technical Report of IEICE, ISEC2000-6 (2000).

3. P. Barreto, V. Rijmen, J. Nakahara Jr., B. Preneel, J. Vandewalle and H. Kim. Improved Square Attacks against Reduced-Round Hierocrypt. Proceedings of Fast Software Encryption (to appear in LNCS by Springer-Verlag) (2001) 173-182.

4. E. Biham. Cryptanalysis of Ladder-DES. Fast Software Encryption, LNCS 1267, Springer-Verlag (1997) 134-138.

5. Camellia Home Page. http://info.isl.ntt.co.jp/camellia/.

6. J. Daemen, L. R. Knudsen and V. Rijmen. The Block Cipher SQUARE. Fast Software Encryption, LNCS 1267, Springer-Verlag (1997) 149-165.

7. J. Daemen and V. Rijmen. AES Proposal: Rijndael (Document version 2). AES Submission (1999).

8. N. Ferguson, J. Kelsey, S. Lucks, B. Schneier, M. Stay, D. Wagner and D. Whiting. Improved Cryptanalysis of Rijndael. Fast Software Encryption, LNCS 1978, Springer-Verlag (2000) 213-230.

9. C. D'Halluin, G. Bijnens, V. Rijmen and B. Preneel. Attack on the Six Rounds of CRYPTON. Fast Software Encryption, LNCS 1636, Springer-Verlag (1999) 46-59.

10. Y. He and S. Qing, Square Attack on Reduced Camellia Cipher. ICICS 2001, LNCS 2229, Springer-Verlag (2001) 238-245.

11. T. Kawabata and T. Kaneko. A Study on Higher Order Differential Attack of Camellia. the 2nd open NESSIE workshop (2001).

12. M. Kanda and T. Matsumoto. Security of Camellia against Truncated Differential Cryptanalysis. Proceedings of Fast Software Encryption (to appear in LNCS by Springer-Verlag) (2001) 298-312.

13. K. Kanda, S. Moriai, K. Aoki, H. Ueda, M. Ohkubo, Y. Takashima, K. Ohta and T. Matsumoto. A New 128-bit Block Cipher E2. Technical Report ISEC98-12, The Institute of Electronics, Information and Communication Engineers. (1998).

14. L. R. Knudsen. Analysis of Camellia. a contribution for ISO/IEC JTC1 SC27. http://info.isl.ntt.co.jp/camellia/Publications/knudsen.ps (2000).

15. S. Lee, S. Hong, S. Lee, J. Lim and S. Yoon. Truncated Differential Cryptanalysis of Camellia. ICISC 2001, (to appear in LNCS by Springer-Verlag) (2001).

16. S. Lucks. Attacking Seven Rounds of Rijndael under 192-bit and 256-bit Keys. Proceedings of 3rd AES Conference (2000).

17. S. Lucks. The Saturation Attack - a Bait for Twofish. Proceedings of Fast Software Encryption (to appear in LNCS by Springer-Verlag) (2001) 1-15.

18. M. Matsui. New Block Encryption Algorithm MISTY. Fast Software Encryption, LNCS 1267, Springer-Verlag (1997) 54-68.

19. M. Sugita, K. Kobara and H. Imai, Security of Reduced Version of the Block Cipher Camellia against Truncated and Impossible Differential Cryptanalysis. ASIACRYPT 2001, LNCS 2248, Springer-Verlag (2001) 193-207.

20. Y. L. Yin. A Note on the Block Cipher Camellia. a contribution for ISO/IEC JTC1 SC27. http://info.isl.ntt.co.jp/camellia/Publications/yiqun.ps (2000). 